\title{
Screening for depressive symptoms among older adults in Taiwan: Cutoff of a short form of the Center for Epidemiologic Studies Depression Scale
}

\author{
Ken-Fu Chang, Li-Jen Weng ${ }^{*}$ \\ Department of Psychology, National Taiwan University, Taipei, Taiwan; ${ }^{*}$ Corresponding Author: ljweng@ntu.edu.tw
}

Received 10 January 2013; revised 15 February 2013; accepted 25 February 2013

\begin{abstract}
Screening for elders at risk for depression is crucial for promoting mental health in later life. The present study investigates the cutoff score of a short form of the CES-D for screening of depressive symptoms in the elderly population of Taiwan. This particular short form of CES-D is repeatedly used in the Taiwan Longitudinal Study on Aging (TLSA), a national survey of representative older adults in Taiwan, and is therefore referred to as the TLSA form. Data collected from five waves of TLSA were analyzed in this study. Participant responses repeatedly measured in 1989 and 1993 were used to identify a cutoff for the TLSA form of the CES-D with sensitivity and specificity using the cutoff of 4 on the Boston form of the CES-D as the criterion. Longitudinal data from the years 1996, 1999, and 2003 were used to validate the proposed cutoff by comparing the prevalence rates for depressive symptoms among older Taiwanese estimated from the identified cutoff and reported in previous studies. The score of 10 on the TLSA form of the CES-D was suggested, yielding a specificity of 0.93 and a sensitivity of approximately 0.96 . The estimated prevalence rates for depressive symptoms among Taiwanese elders based on the suggested cutoff were between $18.9 \%$ and $23.7 \%$, which are similar to previously reported rates. The cutoff score of 10 on the TLSA form of the CES-D is therefore recommended for screening depressive symptoms among older adults in Taiwan.
\end{abstract}

Keywords: CES-D; Cutoff; Depressive Symptoms; Older Adults; Screening; Taiwan Longitudinal Study on Aging

\section{INTRODUCTION}

Depression is one of the most common mental health problems in later life. Yet, depression and depressive symptoms in older adults are often under-identified by both clinical professionals and laypersons. Depressive symptoms can be mistaken as normal aging processes and downplayed by older people themselves [1]. These symptoms may also be neglected because the syndromes manifested among elders can differ from those described in the Diagnostic and Statistical Manual [2]. Nevertheless, it is highly impractical to administer clinical diagnostic interviews to the entire old population to screen for probable depressed elders due to the high cost required. Instead, a convenient screening instrument would be of supreme value [3]. A short form of the Center for Epidemiologic Studies Depression Scale (CES-D Scale) [4] has been frequently used in national surveys of elders in Taiwan and can serve as an effective device. Because the items of this short form of the CES-D differ from those of other previously developed short forms, a cutoff score must be determined. To the best of our knowledge, no research has established the cutoff score for this widely used scale in Taiwan. Therefore, the present study is designed to identify a cutoff score for this short form of the CES-D to screen for elders at risk for depression so that further diagnostic interviews and necessary assistance can be provided in a timely manner.

The Taiwan Longitudinal Study on Aging (TLSA) is a nationally representative survey that was designed to examine the impact of socioeconomic development on the physical and emotional health of older adults in Taiwan. Face-to-face interviews have been conducted by trained interviewers since 1989 under the sponsorship of the Bureau of Health Promotion at the Taiwan Department of Health. The TLSA sample, which was drawn from the household register, is representative of contemporary institutionalized and non-institutionalized Taiwanese older adults aged 60 years and over [5]. The representativeness of the sample ensures the appropriateness of the statistical inferences made and secures the generalizability of the research findings. In addition, the longitudinal TLSA surveys were repeatedly conducted in 
1989, 1993, 1996, 1999, 2003, and 2007, providing rich information on Taiwanese elders over a period of 18 years.

Modified CES-D scales have been adopted in the TLSA to evaluate the depressive symptoms of the interviewees. The original 20-item CES-D scale was developed by Radloff for studies of the epidemiology of depressive symptomatology in general populations [4]. The outstanding psychometric properties of the scale among general populations have been demonstrated in many studies [6]. Notwithstanding its popularity in most settings, the CES-D presents potential problems for older respondents. Older respondents may perceive the length of time needed to complete the scale to be burdensome, consider the questions emotionally stressful, or be confused by the 4 - point response format [7]. Thus, several modified short forms, such as the Andresen form [8], Iowa form, and Boston form [9], have been developed.
However, as shown in Table 1, the items continually used in the TLSA differ from those of other short forms of the CES-D. Among the original 20 items, 10 of them have been repeatedly used across waves of the TLSA and in other national studies in Taiwan (e.g., the National Health Interview Survey, NHIS; the Social Environment and Biomarkers of Aging Study, SEBAS). This 10-item short form of the CES-D is thus called the TLSA form. A study of the cutoff for this particular form of the CES-D is needed to utilize the TLSA to promote the mental health of older citizens in Taiwan.

A number of studies have supported the use of the CES-D as a screening device for probable depression [10, 11]. To be qualified as a screening instrument, a test should demonstrate both high sensitivity and high specificity [12]. Sensitivity refers to the probability that a person who has or will develop the illness under study will show a positive result on the test. Specificity repre-

Table 1. Items of the original CES-D included in TLSA and several short forms of the CES-D.

\begin{tabular}{|c|c|c|c|c|c|c|c|}
\hline Items of the Original CES-D & $\begin{array}{l}\text { TLSA } \\
1989\end{array}$ & $\begin{array}{l}\text { TLSA } \\
1993\end{array}$ & $=$ & $\begin{array}{l}\text { Iowa } \\
\text { Form }\end{array}$ & $\begin{array}{l}\text { TLSA } \\
\text { Form }\end{array}$ & $\begin{array}{l}\text { Boston } \\
\text { Form }\end{array}$ & $\begin{array}{l}\text { Andresen } \\
\text { Form }\end{array}$ \\
\hline I felt everything I did was an effort. & $\mathrm{X}$ & & $\mathrm{X}$ & & $\mathrm{X}$ & $\mathrm{X}$ & $\mathrm{X}$ \\
\hline My sleep was restless. & $\mathrm{X}$ & & $\mathrm{X}$ & & $\mathrm{X}$ & $\mathrm{X}$ & $\mathrm{X}$ \\
\hline I could not get "going". & $\mathrm{X}$ & & $\mathrm{X}$ & & $\mathrm{X}$ & $\mathrm{X}$ & $\mathrm{X}$ \\
\hline I did not feel like eating; my appetite was poor. & $\mathrm{X}$ & & $\mathrm{X}$ & & $\mathrm{X}$ & & \\
\hline I was bothered by things that don't usually bother me. & $\mathrm{X}$ & & & & & & $\mathrm{X}$ \\
\hline I had trouble keeping my mind on what I was doing. & $\mathrm{X}$ & & & & & & $\mathrm{X}$ \\
\hline I talked less than usual. & $\mathrm{X}$ & & & & & & \\
\hline I felt depressed. & $\mathrm{X}$ & & $\mathrm{X}$ & & $\mathrm{X}$ & $\mathrm{X}$ & $\mathrm{X}$ \\
\hline I felt lonely. & $\mathrm{X}$ & & $\mathrm{X}$ & & $\mathrm{X}$ & $\mathrm{X}$ & $\mathrm{X}$ \\
\hline I felt sad. & $\mathrm{X}$ & & $\mathrm{X}$ & & $\mathrm{X}$ & $\mathrm{X}$ & \\
\hline I felt I could not shake off the blues. & $\mathrm{X}$ & & & & & & \\
\hline I felt fearful. & $\mathrm{X}$ & & & & & & $\mathrm{X}$ \\
\hline I thought my life had been a failure. & $\mathrm{X}$ & & & & & & \\
\hline \multicolumn{8}{|l|}{ I had crying spells. } \\
\hline People were unfriendly. & $\mathrm{X}$ & & $\mathrm{X}$ & & $\mathrm{X}$ & $\mathrm{X}$ & \\
\hline I felt that people disliked me. & $\mathrm{X}$ & & $\mathrm{X}$ & & & $\mathrm{X}$ & \\
\hline I was happy. ${ }^{\text {a }}$ & $\mathrm{X}$ & & $\mathrm{X}$ & & $\mathrm{X}$ & $\mathrm{X}$ & $\mathrm{X}$ \\
\hline I enjoyed life. ${ }^{a}$ & $\mathrm{X}$ & & $\mathrm{X}$ & & $\mathrm{X}$ & $\mathrm{X}$ & \\
\hline I felt hopeful about the future. ${ }^{a}$ & & & & & & & $\mathrm{X}$ \\
\hline \multicolumn{8}{|l|}{ I felt as good as other people. ${ }^{a}$} \\
\hline Total number of items & 17 & & 11 & & 10 & 10 & 10 \\
\hline
\end{tabular}

CES-D = the Center for Epidemiologic Studies Depression Scale; TLSA = the Taiwan Longitudinal Study on Aging. a. Positive items. Items of the original CES-D included in TLSA surveys and each short form of the CES-D were indicated by an X. 
sents the probability that a person who does not have the illness under study will obtain a negative result on the test. Commonly, the receiver operator characteristic (ROC) curve is used to examine the sensitivity and specificity at different cutoff scores on the screening instrument. The area under the ROC curve (AUC) represents the degree of correct classification with the screening tool. A larger AUC indicates a more efficient screening device [13].

Various cutoff scores for forms of the CES-D have been proposed based on different criteria for identifying probable depression. For the original CES-D, Radloff proposed a cutoff score of 16 for Caucasian samples above 18 years old, yielding a sensitivity of 0.70 [4]. However, this cutoff was proposed for the general population rather than for elders in particular [4]. Research has shown that the manifestation of depression in elders, who generally display more somatic symptoms and cognitive impairments, differs from that in younger adults [14]. Accordingly, the cutoff for the general population may not be suitable for older adults.

Additional cutoffs for the original CES-D have been recommended to classify probable depressive elders [4]. Himmelfarb and Murrell used 20 as the cutoff for elders in the US and obtained a sensitivity of 0.70 [15]. Cheng and Chan used psychiatrist diagnosis over a range of clinically significant states of depression as the criterion and suggested a cutoff of 22 on the full CES-D for older Hong Kong Chinese individuals, yielding a sensitivity of 0.75 , a specificity of 0.57 , and an AUC of 0.68 [16]. Numerous studies have advocated these cutoff scores or suggested other cutoffs (e.g., 25 and 12) [11,17].

Cutoff scores on short forms of the CES-D have also been proposed. Andresen et al. suggested a threshold of 10 on the Andresen form for relatively healthy American adults aged 65 years and over, yielding a kappa coefficient, sensitivity, and specificity of $0.97,0.96$, and 1.00, respectively [8]. Among a Chinese sample aged 70 years and over in Hong Kong, Boey echoed the finding of Andresen et al. and reached the highest kappa coefficient of 0.84 using the cutoff of 10 on the Andresen form [18]. Cheng and Chan advocated a higher cut-point of 12 on the Andresen form for older Hong Kong Chinese, with a sensitivity of 0.76 , a specificity of 0.55 , and an AUC of $0.68[16]$.

The Boston form is another popular short form of the CES-D. Each item on the 10-item Boston form is coded dichotomously, yielding a total score in the range of 0 to 10. Using middle-aged depressed patients who fulfilled the DSM-IV criteria for major depressive disorder [19] as the criterion, Irwin et al. set a cutoff of 4 to achieve a high sensitivity of 0.97 without much loss in specificity (0.84) [7]. They applied this cutoff to identify older adults with major depression and obtained a sensitivity of 1.00 and a specificity of 0.93 . Robison et al. used the criterion for major depression in the Composite International Diagnostic Interview (CIDI) and obtained a sensitivity and specificity of 0.76 and 0.70 , respectively, among middle-aged and older Puerto Rican primary care patients in the US based on the cutoff of 4 recommended by Irwin et al. [7,20] Further, a cut-point of 3 was recommended for the optimal combination of sensitivity (0.84) and specificity (0.64).

Although numerous cutoff scores for forms of the CES-D have been proposed, none have been suggested for the TLSA form used in Taiwan longitudinally. However, research studies have used a cutoff of 10 for the TLSA form of the CES-D with no empirical evidence provided for its appropriateness (e.g., the Bureau of Health Promotion, Department of Health [5]; Ku et al. [21]). Therefore, the present study was designed to empirically identify a possible cutoff score for the TLSA form of the CES-D as a screening instrument for depressive symptoms among Taiwanese older adults.

\section{METHODS}

\subsection{Sample}

The initial sample of the TLSA, which has been collected since 1989, and the expanded sample set, which was added in 1996, were analyzed in this study. To focus on the old population in Taiwan, only participants aged 60 years and over at the time of data collection were included. Participant responses provided by proxy were excluded because depressive symptoms should be directly reported by respondents. Data with missing responses on any item of the Boston or TLSA form of the CES-D were also excluded. The sample analyzed consisted of 3797, 2930, 3281, 3213, and 3091 individuals in the year of 1989, 1993,1996, 1999, and 2003, respectively. The 1989/1993 data were used to identify a potential cutoff for the TLSA form of the CES-D, and the data from the other three years were used to validate the proposed cutoff score.

\subsection{Instruments}

Table 1 shows the CES-D items that were adopted in each wave of the TLSA and in other short forms of the CES-D. The TLSA form of the CES-D includes items that have been used in the TLSA since 1996. In 1989 and 1993, respondents were asked to rate the frequency with which they experienced the described situations or feelings in the past week on a 4-point scale (not at all $=0$; rarely [less than one day $]=1$; sometimes $[1-4$ days $]=2$; often or chronically [5 - 7 days] $=3$ ). In 1996, the frequency used on the scale was altered without explanation (not at all $=0$; rarely [one day] $=1$; sometimes $[2-3$ 
days $]=2$; often or chronically [over 4 days] $=3$ ). Positive items, as marked in Table $\mathbf{1}$, were reversely coded. Higher scores on the scale represented more depressive symptoms.

\subsection{Data Analysis}

All the items included on the Boston form were assessed in the 1989 and 1993 surveys of the TLSA. Therefore, the 1989/1993 data were used to identify a cutoff for the TLSA form of the CES-D. The procedures proposed by Kohout et al. were followed to convert responses on the original 4-point scale to the dichotomous scores of the 10 items on the Boston form of the CES-D [9]. Answers of 0 or 1 on the 4-point scale were coded as 0 , representing "No descriptive symptoms revealed" on the Boston form, and responses of 2 or 3 were collapsed to form the response category of 1 , representing "Much of the time" on the Boston form.

For the dichotomously coded Boston form of the CES$\mathrm{D}$, Irwin et al. recommended a cutoff of 4 and Robison et al. suggested a cutoff of 3 [7,20]. Separate ROC curves were obtained with the cutoffs of 3 and 4 against various scores on the TLSA form of the CES-D. The confidence intervals of the AUCs were computed to select the curve that led to a better overall performance, as indicated by a larger AUC [22]. Subsequently, the cutoff score on the TLSA form was identified by maximizing the combination of sensitivity and specificity. To examine the classification agreement between the Boston form and the TLSA form, as in Andresen et al. [8], the kappa coefficients [23] were computed for different cutoffs. The kappa coefficient is a chance-corrected measure of agreement, with values greater than 0.75 representing excellent agreement beyond chance.

With the initial sample and the added sample appropriately weighted, the $1996 / 1999 / 2003$ data were used to estimate the prevalence rates for depressive symptoms among elder Taiwanese based on the identified cutoff score of the TLSA form. These prevalence rates were then compared to those found in previous studies to evaluate the appropriateness of the suggested cutoff score. All the analyses were performed using SAS version 8.0 (SAS Institute, Cary, NC, USA).

\section{RESULTS}

Table 2 presents the characteristics of the samples used in this study. The samples had slightly more males than females. Approximately half of the samples were from respondents who were young-old (65 - 74 years) and had received a formal education for less than 6 years. The majority of the elders lived with spouses or partners, and one-quarter of the participants were widowed. Within each wave of the TLSA data analyzed, the par- ticipants who were included in the present study were younger than those who were excluded. However, no difference was found in terms of gender, educational level, or marital status.

ROC curves based on the cutoffs of 3 and 4 on the Boston form against various scores on the TLSA form of the CES-D were conducted using the 1989/1993 TLSA data. As expected, the AUC from the cutoff of 4 was larger than that from the cutoff of 3 in both years. Therefore, only the ROC curves based on the cutoff score of 4 are presented in Figure 1. The 95\% confidence intervals of the AUC for the ROC curves for 1989 and 1993 were $(0.986,0.991)$ and $(0.987,0.992)$, respectively. These curves were used to identify the cutoff for the TLSA form of the CES-D based on sensitivity and specificity.

For both years, a cutoff score of 10 (i.e., 9/10 in Table 3) was recommended on the basis of its high specificities and sensitivities, short distances from the upper left corner of the graph to the ROC curves, and satisfying kappa coefficients, as shown in Table 3 . With the suggested cutoff score of 10, the prevalence rates for depressive symptoms among older adults in Taiwan were $23.7 \%$, $20.9 \%$, and $18.9 \%$ for years 1996,1999 , and 2003 , respectively, as shown at the bottom of Table 2 . These estimated prevalence rates fell in the range of those obtained in previous studies $34.8 \%$ for suburban samples in $\mathrm{Lu}$ et al.; $18.5 \%, 20.3 \%$, and $25.8 \%$ for rural, semiurban, and urban samples, respectively, in Chong et al.; $12.8 \%$ and $20.1 \%$ for rural and urban samples in Chiu et al.; $27.5 \%$ in Tsai et al.) [24-27].

\section{DISCUSSION}

This study aimed to identify a cutoff score for the 10item TLSA form of the CES-D to screen for depressive symptoms among older adults in Taiwan. The TLSA form of the CES-D deserves research attention for two reasons. First, this particular short form of the CES-D differs from other short forms of the CES-D under study. Second, the TLSA short form has been frequently used in national surveys in Taiwan. A cutoff for this widely used short form will be of great value for mental health promotion in Taiwan. Using the cutoff of 4 on the Boston form of the CES-D as the criterion [7], the cutoff of 10 was proposed as the tentative cutoff score for the TLSA form of the CES-D on the basis of the ROC curves. The suggested cutoff was validated with prevalence rates that were estimated in the present study and obtained in previous studies.

Although the prevalence rates that were estimated in the present study were comparable to those in previous studies, the prevalence rates for depressive symptoms varied across studies. Prevalence rate estimates could be affected by numerous factors [28], including the defini- 
Table 2. Characteristics of the analyzed samples.

\begin{tabular}{|c|c|c|c|c|c|c|c|c|c|c|c|}
\hline & \multicolumn{11}{|c|}{ Survey Year in TLSA } \\
\hline & \multirow{2}{*}{1989} & \multirow{2}{*}{1993} & \multicolumn{3}{|c|}{1996} & \multicolumn{3}{|c|}{1999} & \multicolumn{3}{|c|}{2003} \\
\hline & & & A & B & All & A & B & All & A & B & All \\
\hline$N$ & 3797 & 2930 & 2377 & 904 & & 2052 & 1161 & & 1463 & 1628 & \\
\hline Male (\%) & 57.5 & 56.9 & 56.0 & 53.1 & 54.7 & 55.4 & 51.9 & 53.4 & 54.6 & 50.2 & 51.5 \\
\hline \multicolumn{12}{|l|}{ Age $(\%)$} \\
\hline$\leq 64$ & 37.5 & 6.6 & 0.0 & 69.5 & 31.6 & 0.0 & 53.0 & 29.3 & 0.0 & 40.7 & 28.9 \\
\hline $65-74$ & 47.0 & 69.6 & 66.4 & 30.5 & 50.1 & 47.8 & 47.0 & 47.4 & 9.8 & 59.2 & 44.8 \\
\hline $75-84$ & 14.3 & 21.6 & 30.3 & 0.0 & 16.5 & 45.9 & 0.0 & 20.5 & 80.7 & 0.1 & 23.6 \\
\hline$\geq 85$ & 1.3 & 2.2 & 3.3 & 0.0 & 1.8 & 6.3 & 0.0 & 2.8 & 9.4 & 0.0 & 2.8 \\
\hline \multicolumn{12}{|l|}{ Education (\%) } \\
\hline Illiterate & 40.2 & 39.5 & 37.7 & 26.4 & 32.6 & 36.2 & 28.1 & 31.7 & 33.6 & 27.7 & 29.4 \\
\hline$\leq 6$ Years & 40.4 & 41.4 & 41.9 & 51.6 & 46.3 & 42.4 & 50.7 & 47.0 & 43.0 & 49.5 & 47.6 \\
\hline $7-12$ Years & 14.3 & 14.1 & 14.8 & 17.4 & 16.0 & 15.8 & 16.5 & 16.1 & 17.1 & 17.0 & 17.0 \\
\hline$>12$ Years & 5.1 & 5.1 & 5.6 & 4.7 & 5.2 & 5.7 & 4.8 & 5.2 & 6.3 & 5.8 & 5.9 \\
\hline \multicolumn{12}{|l|}{ Marital Status (\%) } \\
\hline With Spouse/Partner & 63.8 & 63.3 & 60.2 & 76.8 & 67.7 & 56.1 & 77.6 & 68.0 & 51.2 & 76.8 & 69.4 \\
\hline Widowed & 26.8 & 32.2 & 34.5 & 15.2 & 25.7 & 38.8 & 16.5 & 26.5 & 44.4 & 19.1 & 26.5 \\
\hline Separated/Divorced & 5.7 & 1.0 & 1.6 & 3.2 & 2.4 & 1.6 & 2.8 & 2.3 & 1.5 & 2.5 & 2.2 \\
\hline Never been married & 3.7 & 3.5 & 3.6 & 4.9 & 4.2 & 3.5 & 3.0 & 3.2 & 2.9 & 1.6 & 2.0 \\
\hline $\begin{array}{l}\text { Prevalence Rate for } \\
\text { Depressive Symptoms }\end{array}$ & & & $26.8 \%$ & $20.0 \%$ & $23.7 \%$ & $26.0 \%$ & $16.8 \%$ & $20.9 \%$ & $21.2 \%$ & $18.0 \%$ & $18.9 \%$ \\
\hline
\end{tabular}

The total percentage of some categories may not equal 100 due to rounding. TLSA = the Taiwan Longitudinal Study on Aging; A = Sample A, the TLSA sample collected since 1989; B = Sample B, the TLSA sample added in 1996; All = the pooled sample of Sample A and Sample B with weights.

Table 3. The performance of selected cutoffs on the TLSA form of the CES-D in 1989 and 1993.

\begin{tabular}{|c|c|c|c|c|c|c|}
\hline Year & Cutoff & Spec & Sens & Spec + Sens & PPV & Kappa \\
\hline \multirow[t]{5}{*}{1989} & $7 / 8$ & 0.810 & 0.998 & 1.808 & 0.508 & 0.58 \\
\hline & $8 / 9$ & 0.887 & 0.989 & 1.876 & 0.634 & 0.72 \\
\hline & $9 / 10$ & 0.933 & 0.963 & 1.896 & 0.740 & 0.80 \\
\hline & $10 / 11$ & 0.966 & 0.885 & 1.851 & 0.838 & 0.83 \\
\hline & $11 / 12$ & 0.986 & 0.795 & 1.781 & 0.920 & 0.83 \\
\hline \multirow[t]{5}{*}{1993} & $7 / 8$ & 0.812 & 0.998 & 1.810 & 0.572 & 0.63 \\
\hline & $8 / 9$ & 0.885 & 0.990 & 1.875 & 0.684 & 0.75 \\
\hline & $9 / 10$ & 0.933 & 0.966 & 1.899 & 0.785 & 0.83 \\
\hline & $10 / 11$ & 0.967 & 0.881 & 1.848 & 0.871 & 0.84 \\
\hline & $11 / 12$ & 0.985 & 0.817 & 1.802 & 0.930 & 0.84 \\
\hline
\end{tabular}

TLSA = the Taiwan Longitudinal Study on Aging; CES-D = the Center for Epidemiologic Studies Depression Scale; Spec = Specificity; Sens = Sensitivity; $\mathrm{PPV}=$ Positive Predictive Value. 


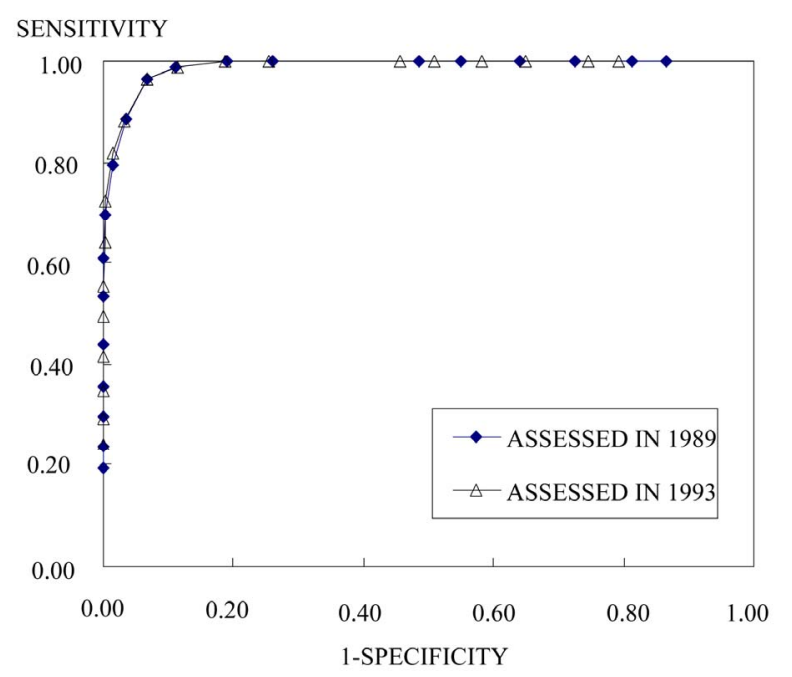

Figure 1. Receiver operator characteristic curves for the TLSA form of the CES-D with cutoff 4 on the Boston form as the criterion. The curves assessed in 1989 and 1993 were overlapping with the $95 \%$ confidence intervals of the AUC being $(0.986,0.991)$ and $(0.987,0.992)$, respectively.

tion of depressive cases, the nature of screening instruments, the cutoff adopted, and the characteristics of the samples studied. Therefore, it is essential to further validate the cutoff score of 10 for the TLSA form of the CES-D with clinically diagnosed depressed patients. The Bureau of Health Promotion at the Department of Health in Taiwan and $\mathrm{Ku}$ et al. [5,21] employed the cutoff of 10 . Nevertheless, to the best of our knowledge, the present study was the first to provide empirical findings in support of the use of 10 as the cutoff on the TLSA form of the CES-D. This suggested cutoff can be used as a tentative criterion to screen for older Taiwanese with severe depressive symptoms so that necessary professional assessment and care can be provided to the elders who may be at risk for depression in a timely manner.

\section{CONCLUSION}

A cutoff score of 10 is recommended for the TLSA form of CES-D to screen for elders at risk for depression in Taiwan. This cutoff yielded prevalence rates of depressive symptoms similar to previously reported ones. With the widely use of this particular short form of CES$\mathrm{D}$ in Taiwan, the suggested cutoff can be used to identify older Taiwanese who may need comprehensive assessment and care for mental health. This tentative cutoff can further be validated with clinically diagnosed depressed elders to enhance the utility of the TLSA form of CES-D for promoting mental health in Taiwan.

\section{ACKNOWLEDGEMENTS}

The authors would like to thank the Bureau of Health Promotion at the Department of Health in Taiwan for the permission to analyze the data from the Taiwan Longitudinal Study on Aging (TLSA). The descriptions and conclusions herein do not represent the viewpoint of the Bureau. The authors also wish to thank Dr. Chi Chiao of the National Yang-Ming University and Dr. Chung-Ping Cheng of the National Cheng-Kung University for their stimulating discussions and valuable suggestions on this work.

\section{REFERENCES}

[1] Mui, A.C. and Yang, P.S. (2005) The foundations of gerontological social work practice in Taiwan. YehYeh Publishing House, Taipei.

[2] Small, G.W. (1998) Treatment of geriatric depression. Depression and Anxiety, 8, 32-42. doi:10.1002/(SICI)1520-6394(1998)8:1+<32::AID-DA6> 3.0.CO;2-J

[3] Katona, C.L.E. (1994) Depression in old age. Wiley, New York.

[4] Radloff, L.S. (1977) The CES-D scale: A self-report depression scale for research in the general population. Applied Psychological Measurement, 1, 385-401. doi: $10.1177 / 014662167700100306$

[5] Bureau of Health Promotion, Department of Health (2006) 2003 Survey of Health and Living Status of the Middle Aged and Elderly in Taiwan Survey Report. Bureau of Health Promotion, Department of Health, Taichung.

[6] Roberts, R.E. and Vernon, S.W. (1983) The Center for Epidemiologic Studies Depression Scale: Its use in a community sample. American Journal of Psychiatry, 140, 41-46.

[7] Irwin, M., Artin, K.H. and Oxman, M.N. (1999) Screening for depression in the older adult: Criterion validity of the 10-item Center for Epidemiological Studies Depression Scale (CES-D). Archives of Internal Medicine, 159, 1701-1704. doi:10.1001/archinte.159.15.1701

[8] Andresen, E.M., Malmgren, J.A., Carter, W.B. and Patrick, D.L. (1994) Screening for depression in well older adults: Evaluation of a short form of the CES-D (Center for Epidemiologic Studies Depression Scale). American Journal of Preventive Medicine, 10, 77-84.

[9] Kohout, F.J., Berkman, L.F., Evans, D.A. and CornoniHuntley, J. (1993) Two shorter forms of the CES-D depression symptoms index. Journal of Aging and Health, 5, 179-193. doi:10.1177/089826439300500202

[10] Myers, J.K. and Weissman, M.M. (1980) Use of a selfreport symptom scale to detect depression in a community sample. American Journal of Psychiatry, 137, 10811084.

[11] Haringsma, R., Engels, G.I., Beekman, A.T. and Spinhoven, P. (2004) The criterion validity of the Center for Epidemiological Studies Depression Scale (CES-D) in a sample of self-referred elders with depressive symptomatology. International Journal of Geriatric Psychiatry, 19, 558-563. doi: $10.1002 /$ gps. 1130

[12] Lewinsohn, P.M., Seeley, J.R., Roberts, R.E. and Allen, N.B. (1997) Center for Epidemiologic Studies Depression 
Scale (CES-D) as a screening instrument for depression among community-residing older adults. Psychology and Aging, 12, 277-287. doi:10.1037/0882-7974.12.2.277

[13] Hanley, J.A. and McNeil, B.J. (1982) The meaning and use of the area under receiver operating characteristic (ROC) curve. Radiology, 143, 29-36.

[14] Sheehan, B. and Banerjee, S. (1999) Review: Somatization in the elderly. International Journal of Geriatric Psychiatry, 14, 1044-1049. doi:10.1002/(SICI)1099-1166(199912)14:12<1044::AIDGPS55>3.0.CO;2-0

[15] Himmelfarb, S. and Murrell, S.A. (1983) Reliability and validity of five mental health scales in older persons. Journal of Gerontology, 38, 333-339. doi:10.1093/geronj/38.3.333

[16] Cheng, S.T. and Chan, A.C.M. (2005) The Center for Epidemiologic Studies Depression Scale in older Chinese: Thresholds for long and short forms. International Journal of Geriatric Psychiatry, 20, 465-470. doi:10.1002/gps.1314

[17] Waston, L.C., Lewis, C.L., Kistler, C.E., Amick, H.R. and Boustani, M. (2004) Can we trust depression screening instruments in healthy "old-old" adults? International Journal of Geriatric Psychiatry, 19, 278-285. doi: $10.1002 /$ gps. 1082

[18] Boey, K.W. (1999) Cross-validation of a short form of the CES-D in Chinese elderly. International Journal of Geriatric Psychiatry, 14, 608-617. doi:10.1002/(SICI)1099-1166(199908)14:8<608::AID-G PS991>3.0.CO;2-Z

[19] American Psychiatric Association (1994) Diagnostic and statistical manual of mental disorders. 4th Edition, American Psychiatric Association, Washington DC.

[20] Robison, J., Gruman, C., Gaztambide, S. and Blank, K. (2002) Screening for depression in middle-aged and older
Puerto Rican primary care patients. Journals of Gerontology Series A: Biological Sciences and Medical, 57, M308-M314. doi:10.1093/gerona/57.5.M308

[21] Ku, P.W., Fox, K.R. and Chen, L.J. (2009) Physical activity and depressive symptoms in Taiwanese older adults: A seven-year follow-up study. Preventive Medicine, 48, 250-255. doi:10.1016/j.ypmed.2009.01.006

[22] SAS Institute, Inc. (2001) Sample 25017: Nonparametric comparison of areas under correlated ROC curves. http://support.sas.com/kb/25/017.html

[23] Fleiss, J.L. (1981) Statistical methods for rates and proportions. 2nd Edition, Wiley \& Sons, New York.

[24] Lu, C.H., Liu, C.Y. and Yu, S. (1998) Depressive disorders among the Chinese elderly in a suburban community. Public Health Nursing, 15, 196-200. doi:10.1111/j.1525-1446.1998.tb00339.x

[25] Chong, M.Y., Tsang, H.Y., Chen, C.S., et al. (2001) Community study of depression in old age in Taiwan: Prevalence, life events and socio-demographic correlates. British Journal of Psychiatry, 178, 29-35. doi:10.1192/bjp.178.1.29

[26] Chiu, H.C., Chen, C.M., Huang, C.J. and Mau, L.W. (2005) Depressive symptoms, chronic medical conditions and functional status: A comparison of urban and rural elders in Taiwan. International Journal of Geriatric Psychiatry, 20, 635-644. doi:10.1002/gps.1292

[27] Tsai, Y.F., Yeh, S.H. and Tsai, H.H. (2005) Prevalence and risk factors for depressive symptoms among communitydwelling elders in Taiwan. International Journal of Geriatric Psychiatry, 20, 1097-1102. doi: $10.1002 /$ gps. 1413

[28] Beekman, A.T., Copeland, J.R. and Prince, M.J. (1999) Review of community prevalence of depression in later life. British Journal of Psychiatry, 174, 307-311. doi:10.1192/bjp.174.4.307 